\title{
Foreword - The importance of a healthy microbiota in the era of COVID-19
}

\section{A year after the COVID-19 pandemic}

At the start of 2020 we were thrilled to have reached 10 years of Beneficial Microbes! Little did we know that soon after Europe and the rest of the world (with Asia already earlier) would be in lock-down due to COVID-19. It has been a strange year. And now, at the start of 2021, the excitement of having a vaccine is tempered by the fact that everywhere mutants of the virus pop up. Although this was likely to occur, as also the influenza virus keeps mutating, it means that at the moment it is unclear as to whether the current situation of lock-downs and social distancing will remain for a longer period than we had anticipated and hoped for at the end of 2020 when it became clear that several vaccines were efficacious. Some studies have shown a role of the gut microbiota composition in disease severity, together with vitamin D, cholesterol and other factors. It was a hype to write a 'review' on gut microbiota and the effect on COVID-19, and also the board of Beneficial Microbes has received several submissions of socalled reviews on the topic. However, all of them were rejected, as they were mere speculations about how the gut microbiota might affect virus infection and disease severity, without any data whatsoever. However, there are some good studies out there that have shown that a proper gut microbiota may indeed influence disease severity, such as recently reviewed by Kim (2021). All in all, this may not be too surprising for the knowledgeable reader, as they would know that the microbiota plays a role in everything that can be wrong with us!

Traditionally (after 11 years I guess we can call it a tradition) I start the foreword with a brief description of the development of the impact factor (IF). With just 11 years, we are still considered a young journal, and in the beginning of the existence of young journals the IF will fluctuate a little. But over the past couple of years our IF has been on the rise! Last June we reached 3.370 (up from 2.939 in 2019 and 2.310 in 2018). Although I would still like to have a probiotic that interacts with my brain in such a way that I will be able to predict the future (if you don't follow this crazy statement, please read previous forewords), I think it is safe to say (and predict) that by the end of June 2021 (when the new IF will be provided) we will have again reached a higher IF, as the year-to-date score (where the Clarivate year runs from June to June) is currently 3.988 . Still a few more citations to go to reach the magic border of 4 !

This time I also had a look at papers with the highest number of citations. Not surprisingly, the top 10 highest cited papers in Beneficial Microbes are all reviews. And, also not surprisingly, they are all published in the first 4 years of the journal's existence. These had obviously more time to collect citations. The top cited paper is the first paper, in the first issue of the first volume (-)) by Frederique
Chaucheyras-Durand and Henri Durand about 'Probiotics in animal nutrition and health' (Chaucheyras-Durand and Durand, 2010), with 188 citations in the Web of Science Core Collection. It is interesting to note that it took a while before this paper was 'discovered': from the start is has been cited about 5 times/year, but since 2015 this increased to about 25 times/year (Figure 1).

We see that the number of manuscripts being submitted stabilises around 200 and a few per year. This still allows us to be critical about which manuscripts we select for reviewing, because we now have sufficient numbers of manuscripts. In fact, we have decided to increase the number of issues to be published in the last two years to 8 issues/year, which is double the amount that we started with 11 years ago.

The following part is re-iterated from last year, as I believe it is important to note: Authors of manuscripts that are immediately rejected (without a review process) may have noted that we have implemented that the editorial board actually screens each manuscript before it is processed further. This is to protect our reviewers if you like ... We want to prevent that our reviewers get manuscripts that either do not fit in the scope, or are of insufficient merit or 


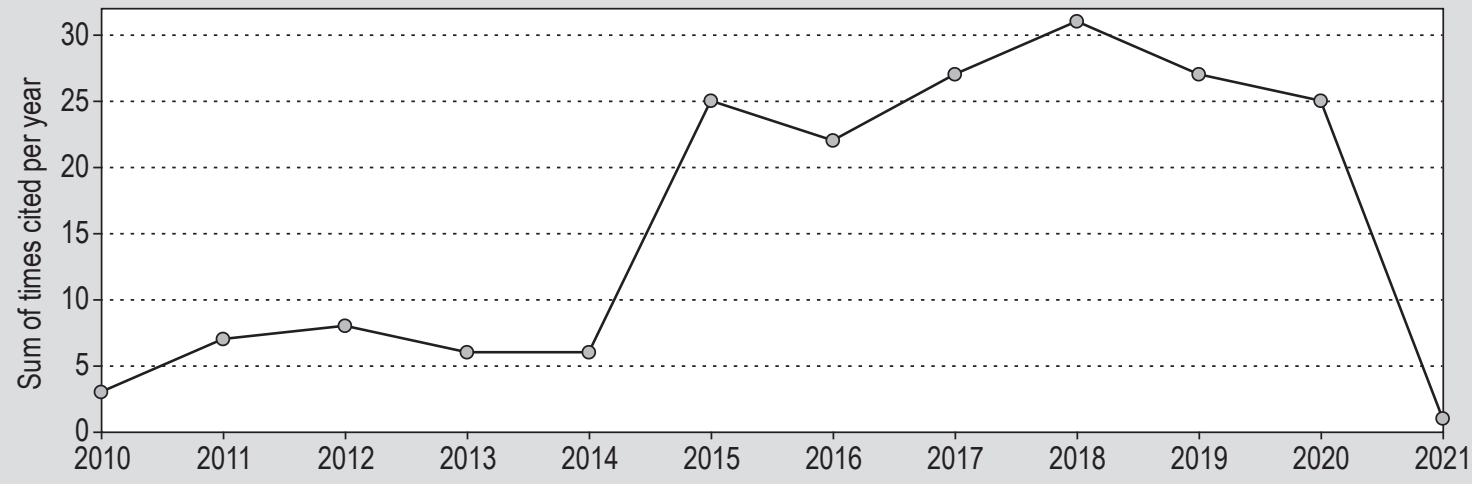

Figure 1. Citations over time for Chaucheyras-Durand and Durand's 'Probiotics in animal nutrition and health', totalling 188 citations.

insufficient breadth to be published in the journal. In this way, we hope to reduce the workload of our reviewers, as we experience that it is still increasingly difficult to find people that are willing and able to review our manuscripts. Sometimes the section editors (SEs) have to invite more than 15 potential reviewers (our record stands at 21!) before they have found two that are willing and able to review a manuscript. Because we do not invite all these $>15$ people at the same time, this sometimes means that some of the manuscripts are in our system for a long period. Part of this is caused also by reviewers that simply do not respond to our invite, which makes it difficult to determine whether it is time to select new potential reviewers, or we should wait a little longer. So, I would urge everyone that reads this to please click on the decline- or unavailable-link if you are not able or not willing to review a particular manuscript for Beneficial Microbes. Hopefully that can reduce the average throughput time in Manuscript Central (MC). By the way, I would like to sincerely thank those reviewers that do this already and especially those that take the time to suggest another potential reviewer. This makes the life of the SEs a lot easier. Also, I want to sincerely thank all reviewers that do accept the invitation to review for our journal. Because of your critical reviews the quality of the papers (when I relate quality of the papers to IF of the journal for reasons of simplicity) has increased over the years.

We are indebted also to our three new and very active and talented SEs: Baltasar Mayo Perez, Devin Rose and Mariya Petrova. Together with Isaac Cann who has been on (the) board from the beginning, they have been dealing with the $>200$ manuscripts and also have been instrumental in the success of the journal, so I'd like to thank them for their efforts here! Moreover, I am indebted to the editorial office for processing all the manuscripts that are accepted and for getting them out as soon as possible, both as e-publication as well as in hard copy, even in these trying times.

\section{Back to science}

Whereas in the other years, I used to peek forward by briefly discussing some of the manuscripts in the corresponding issue in which this foreword is published, this year I would like to look back at what $>10$ years of beneficial microbes has taught us, and how we might learn from this to combat the current global pandemic, or any future ones. Mind you 'beneficial microbes' without capital initial letters. I would like to review the accomplishments in the whole field, not just what is published in our journal.

We all know that the gut microbiota is involved in essentially everything that can be wrong with us, since they interact with us, their host, in many different ways, such as with the immune system and our metabolism. Thus, differences in microbiota composition and/or activity may lead to differences in disease severity when contracting a virus (or other infection). Of course, this is just one of the factors that influences the outcome, with our nutritional status, our own genetic make-up, health status, other diseases we may have, vitamin D levels, cholesterol, etc. contributing as well (Kim, 2021). Several levels of evidence support the use of fermented foods, probiotics and/or prebiotics to promote immunity of both gut and lungs (Antunes et al., 2020; Kim, 2021). Without at forehand being able to predict their efficacy against COVID-19, incorporating probiotics and prebiotics into the diet may help to reduce gut inflammation and to enhance mucosal immunity. That is, on the one hand certain fermented foods and probiotics may deliver viable microbes with the potential to promote (gut or systemic) immunity. Prebiotics, on their side, may enhance gut immunity by selectively stimulating certain resident microbes in the gut (Antunes et al., 2020). This way, beneficial microbes may definitely contribute to COVID severity, and other infectious diseases that may pop up as a global pandemic in the future as well. Of course, not all probiotics are the same and the same holds true for 
prebiotics, and therefore their efficacy must be studied in more detail. Large studies that investigate which is the factor that causes probiotics to modulate our immune system, or structure-function relationships to decipher which prebiotics stimulate which microbes in the colon are required. This will generate tons of data and many correlations between beneficial microbes and disease and disorders, but this ultimately should lead to causal links between certain pro- or prebiotics and health benefits.

The challenge for the coming decade in beneficial microbes research will be developing the bioinformatics tools to cope with the tons of data and to make sense of the exabytes ( 1 million terabytes) or zettabyte ( 1 billion terabyte) of data that are being generated. There are too few experts in this field. Also, there is not enough consensus about the tools developed. Although most people use QIIME2 (Bolyen et al., 2019), including my own research group, other initiatives are also developed, such as Kraken 2 (Lu and Salzberg, 2020; Wood et al., 2019). And here I just mention two of the many bioinformatics pipelines that are being used to investigate just the first step of the link between microbiota and health and disease: microbial composition on the basis of $16 \mathrm{~S}$ rRNA sequencing. But even the sequencing protocols are not aligned between researchers, with some groups sequencing just the V4 region, others $\mathrm{V} 3+\mathrm{V} 4$ (like us), and again others choosing yet other variable regions. No doubt, advances in sequencing technology (if only for the fact that length of sequencing reads may increase and allow high-throughput (almost) full length $16 \mathrm{~S}$ rRNA sequencing) will contribute to consensus tools being used by the scientific community in the future. I hope that our journal will contribute to dissemination of those exiting results.

We, the editorial board, the editorial office and myself, the editor-in-chief, are expecting (predicting) another exiting year (and most likely also decade) for Beneficial Microbes. Stay tuned!

\section{Koen Venema}

Editor-in-chief

\section{References}

Antunes, A.E.C., Vinderola, G., Xavier-Santos, D. and Sivieri, K., 2020. Potential contribution of beneficial microbes to face the COVID-19 pandemic. Food Research International 136: 109577. https://doi. org/10.1016/j.foodres.2020.109577

Bolyen, E., Rideout, J.R., Dillon, M.R., Bokulich, N.A., Abnet, C.C., AlGhalith, G.A., Alexander, H., Alm, E.J., Arumugam, M., Asnicar, F., Bai, Y., Bisanz, J.E., Bittinger, K., Brejnrod, A., Brislawn, C.J., Brown, C.T., Callahan, B.J., Caraballo-Rodriguez, A.M., Chase, J., Cope, E.K., Da Silva, R., Diener, C., Dorrestein, P.C., Douglas, G.M., Durall, D.M., Duvallet, C., Edwardson, C.F., Ernst, M., Estaki, M., Fouquier, J., Gauglitz, J.M., Gibbons, S.M., Gibson, D.L., Gonzalez, A., Gorlick, K., Guo, J., Hillmann, B., Holmes, S., Holste, H., Huttenhower, C., Huttley, G.A., Janssen, S., Jarmusch, A.K., Jiang, L., Kaehler, B.D., Kang, K.B., Keefe, C.R., Keim, P., Kelley, S.T., Knights, D., Koester, I., Kosciolek, T., Kreps, J., Langille, M.G.I., Lee, J., Ley, R., Liu, Y.X., Loftfield, E., Lozupone, C., Maher, M., Marotz, C., Martin, B.D., McDonald, D., McIver, L.J., Melnik, A.V., Metcalf, J.L., Morgan, S.C., Morton, J.T., Naimey, A.T., Navas-Molina, J.A., Nothias, L.F., Orchanian, S.B., Pearson, T., Peoples, S.L., Petras, D., Preuss, M.L., Pruesse, E., Rasmussen, L.B., Rivers, A., Robeson, M.S., 2nd, Rosenthal, P., Segata, N., Shaffer, M., Shiffer, A., Sinha, R., Song, S.J., Spear, J.R., Swafford, A.D., Thompson, L.R., Torres, P.J., Trinh, P., Tripathi, A., Turnbaugh, P.J., Ul-Hasan, S., van der Hooft, J.J.J., Vargas, F., Vazquez-Baeza, Y., Vogtmann, E., von Hippel, M., Walters, W., Wan, Y., Wang, M., Warren, J., Weber, K.C., Williamson, C.H.D., Willis, A.D., Xu, Z.Z., Zaneveld, J.R., Zhang, Y., Zhu, Q., Knight, R. and Caporaso, J.G., 2019. Reproducible, interactive, scalable and extensible microbiome data science using QIIME 2. Nature Biotechnology 37: 852-857. https://doi.org/10.1038/ s41587-019-0209-9

Chaucheyras-Durand, F. and Durand, H., 2010. Probiotics in animal nutrition and health. Beneficial Microbes 1: 3-9. https://doi. org/10.3920/BM2008.1002

Kim, H.S., 2021. Do an altered gut microbiota and an associated leaky gut affect COVID-19 severity? mBio 12: e03022-20. https://doi. org/10.1128/mBio.03022-20

Lu, J. and Salzberg, S.L., 2020. Ultrafast and accurate 16S rRNA microbial community analysis using Kraken 2. Microbiome 8: 124. https://doi.org/10.1186/s40168-020-00900-2

Wood, D.E., Lu, J. and Langmead, B., 2019. Improved metagenomic analysis with Kraken 2. Genome Biology 20: 257. https://doi. org/10.1186/s13059-019-1891-0 
